Journal of Sound and Vibration, 313(3-5), pp. 858-874 (2008).

\title{
Asymptotic equivalence of homogenisation procedures and fine-tuning of continuum theories
}

\author{
A. V. Pichugin ${ }^{a, 1}$, H. Askes ${ }^{b}$ and A. Tyas ${ }^{b}$ \\ ${ }^{a}$ Department of Mathematical Sciences, \\ Brunel University, Uxbridge UB8 3PH, UK; \\ ${ }^{\mathrm{b}}$ Department of Civil and Structural Engineering, \\ University of Sheffield, Sheffield S1 3JD, UK.
}

\begin{abstract}
Long-wave models obtained in the process of asymptotic homogenisation of structures with a characteristic length scale are known to be non-unique. The term nonuniqueness is used here in the sense that various homogenisation strategies may lead to distinct governing equations that usually, for a given order of the governing equation, approximate the original problem with the same asymptotic accuracy. A constructive procedure presented in this paper generates a class of asymptotically equivalent long-wave models from an original homogenised theory. The described non-uniqueness manifests itself in the occurrence of additional parameters characterising the model. A simple problem of long-wave propagation in a regular onedimensional lattice structure is used to illustrate important criteria for selecting these parameters. The procedure is then applied to derive a class of continuum theories for a two-dimensional square array of particles. Applications to asymptotic structural theories are also discussed. In particular, we demonstrate how to improve the governing equation for the Rayleigh-Love rod and explain the reasons for the well-known numerical accuracy of the Mindlin plate theory.
\end{abstract}

Key words: asymptotics, Hermite-Padé approximants, homogenisation, wave propagation, dispersion, Rayleigh-Love rod, Mindlin plate

$\overline{1}$ E-mail: aleksey.pichugin@brunel.ac.uk 


\section{Introduction}

Structures with a characteristic length scale occur in many areas of physics and engineering. The origin of the length scale may be different, say, it may be the typical grain size of a particulate or the thickness of an elastic waveguide; in all cases, these structures naturally lend themselves to continuous modelling using singularly perturbed asymptotic models. The perturbations are invariably based on considering wave fields that vary slowly with respect to the characteristic length scale. The resulting asymptotic models are usually referred to as long-wave theories and the procedure of deriving them from a model with full detail as homogenisation.

Various, sometimes contradicting, requirements on the long-wave theories resulted in a plethora of distinct approaches to asymptotic homogenisation. These approaches are often designed to satisfy certain ad hoc criteria and usually result in models with identical asymptotic accuracy. Attempts at comparing between these models often lead to even greater confusion: where some fail to discriminate the models by their asymptotic accuracy alone, others try to generalise from the performance in particular model problems.

All long-wave theories are, in principle, asymptotic, but they are often used in a purely numerical context. Practitioners are well aware that infinitesimal parameter that enables asymptotic expansion is only finitely small in practice and, sometimes, not very small. And it is not unusual for theoreticians to propose an asymptotic model that becomes ill-posed outside of the intended domain of applicability, which may, for example, make numerical computations unstable. This dichotomy of outlooks resulted in a curious situation when, for example, the most widely used refined plate theory by Mindlin [1] is not even a second order asymptotic of the associated three-dimensional problem. The point is, the practitioners are only concerned with the "approximation" part of the asymptotic approximation and theoreticians tend to ignore it altogether.

In this paper we discuss numerical accuracy and stability of long-wave theories when the small parameter is not very small. This problem has been previously considered for periodically inhomogeneous elastic medium, see [2], where newly-designed variational/asymptotic homogenisation procedure was used to derive strain gradient theories that are both asymptotically consistent and stable. Our approach is different in that it is based on a simple formal procedure capable of generating a class of asymptotically equivalent long-wave theories from a single original homogenised model. Thus, we are shifting focus away from the homogenisation procedure and explore numerical performance of the otherwise equivalent long-wave models. The dispersion relations for the generated classes of asymptotic theories turn out to be related to so-called Hermite-Padé approximants first introduced in Padé's original work but little 
studied since (this area was revived by Shafer [3]).

The described classes of theories are characterised by a number of auxiliary parameters. We use a range of model problems to introduce and discuss several criteria to help specifying these parameters. In particular, we look into ways to ensure numerical stability and physical relevance of the response. In doing so it is necessary to consider the behaviour of asymptotic theories beyond their validity range. The motivation for this is purely pragmatic in that although we cannot attach any physical significance to the results obtained beyond the validity range of the theory, the resulting models are more useful in a purely numerical context. In every considered case the form of the generalised theory makes it also possible to match a few extra terms of the long-wave expansion of the exact dispersion relation. Thus, the newly formulated theories may both be "good" from a numerical point of view and possess extra asymptotic accuracy.

Since all of the considered long-wave theories may feature fourth and higher order spatial derivatives, application of these theories to finite domains requires formulating appropriate boundary conditions. This is an important and complex problem that we felt must be investigated separately. For most of the theories discussed in this paper we identified equivalent sub-classes with modified inertia, see [4], which feature no spatial non-locality and can therefore be used without reformulating the boundary conditions.

\section{Model problem}

Let us illustrate our ideas with the simple model problem first analysed by Newton. Consider a one-dimensional regular array of particles connected by springs, see Figure 1. If the mass of each particle is denoted by $M$ and the stiffness of each spring by $K$, then the motion of the $n$th particle is described by the following equation

$$
M \ddot{u}_{n}=K\left(u_{n-1}-2 u_{n}+u_{n+1}\right), \quad n \in \mathbb{I},
$$

in which the overdot denotes differentiation with respect to time and $u_{n}=$ $u_{n}(t) \equiv u\left(x_{n}, t\right)$ is the longitudinal deflection of the $n$th particle from the equilibrium position $x_{n}=n l$, where $l$ is the interparticle distance.

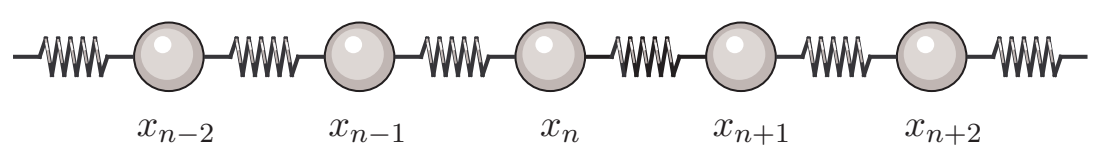

Fig. 1. One-dimensional array of particles connected by springs. 
The steady-state response of the array to a harmonic excitation with angular frequency $\omega$ may be determined by seeking solutions of the form

$$
u_{n}=U e^{\mathrm{i}\left(k x_{n}-\omega t\right)},
$$

with amplitude $U$ and wave number $k$. When solutions (2) are inserted into the governing equation (1), one obtains the dispersion relation for Bloch waves in the array, which may be written as

$$
\bar{\omega}^{2}=2-2 \cos \eta \equiv 4 \sin ^{2} \frac{\eta}{2} \quad \text { or } \quad \eta^{2}=\arccos ^{2}\left(1-\frac{\bar{\omega}^{2}}{2}\right)
$$

where $\bar{\omega}$ is the non-dimensional frequency and $\eta$ the non-dimensional wave number defined as

$$
\bar{\omega}=\frac{\omega}{\Omega}, \quad \Omega=\sqrt{\frac{K}{M}}, \quad \eta=k l .
$$

It is worth noting that all physically meaningful solutions of (3) lie within the first Brillouin zone, i.e. $|\eta| \leqslant \pi$, see e.g. [5]. The described lattice structure is a classical example of low-pass filter, which only allows propagation of waves with frequencies below the cut-off frequency $\bar{\omega}_{c r}=2$. A corresponding dispersion curve may be found in Figure 2.

\subsection{Homogenisation procedure}

The standard homogenisation procedure for equation (1) involves introducing a continuous displacement field $u \equiv u(x, t)$, such that $u\left(x_{n}, t\right)=u_{n}(t)$, and expanding $u_{n \pm 1}(t)$ into Taylor series around $u_{n}(t)$. This enables us to rewrite equation (1) in the following form

$$
\ddot{u}=\Omega^{2} \sum_{n=1}^{\infty} \frac{2}{(2 n) !} \frac{\partial^{2 n} u}{\partial x^{2 n}} l^{2 n},
$$

with the array natural frequency $\Omega$ defined in equation (4). This infinite series representation is exact provided $u$ is smooth enough; it is not, however, convenient for practical purposes. The principal advantage of governing equation (5) is best seen when it is non-dimensionalised according to

$$
x=\xi / k, \quad t=\tau /(\eta \Omega),
$$

which for $u^{*}(\xi, \tau) \equiv \eta^{2} l u(x(\xi, \tau), t(\xi, \tau))$ yields

$$
\frac{\partial^{2} u^{*}}{\partial \tau^{2}}=\sum_{n=1}^{\infty} \frac{2}{(2 n) !} \frac{\partial^{2 n} u^{*}}{\partial \xi^{2 n}} \eta^{2 n-2} .
$$


Let us now consider propagation of long waves, i.e. waves with wavelengths $\lambda \gg l$, so that parameter $\eta=k l \equiv l / \lambda$ becomes small. The dispersion relation (3) may then be expanded into asymptotic series for $\eta \sim \bar{\omega} \rightarrow 0$, yielding

$$
\bar{\omega}^{2}=\eta^{2}-\frac{\eta^{4}}{12}+\frac{\eta^{6}}{360}+O\left(\eta^{8}\right) \quad \text { or } \quad \eta^{2}=\bar{\omega}^{2}+\frac{\bar{\omega}^{4}}{12}+\frac{\bar{\omega}^{6}}{90}+O\left(\bar{\omega}^{8}\right) .
$$

If we assume that differentiation with respect to $\xi$ and $\tau$ does not change asymptotic order of non-dimensional quantities, the series at the right hand side of (7) may also be regarded asymptotic and truncated. In this paper we consider second, fourth, and sixth order theories. This terminology refers to the asymptotic order of the neglected terms ${ }^{2}$. Specifically, these theories have the form

$$
\begin{gathered}
\frac{\partial^{2} u^{*}}{\partial \tau^{2}}=\frac{\partial^{2} u^{*}}{\partial \xi^{2}}+O\left(\eta^{2}\right), \\
\frac{\partial^{2} u^{*}}{\partial \tau^{2}}=\frac{\partial^{2} u^{*}}{\partial \xi^{2}}+\frac{1}{12} \frac{\partial^{4} u^{*}}{\partial \xi^{4}} \eta^{2}+O\left(\eta^{4}\right), \\
\frac{\partial^{2} u^{*}}{\partial \tau^{2}}=\frac{\partial^{2} u^{*}}{\partial \xi^{2}}+\frac{1}{12} \frac{\partial^{4} u^{*}}{\partial \xi^{4}} \eta^{2}+\frac{1}{360} \frac{\partial^{6} u^{*}}{\partial \xi^{6}} \eta^{4}+O\left(\eta^{6}\right) .
\end{gathered}
$$

The asymptotic consistency of (9)-(11) may be evaluated by inserting harmonic wave solutions $U \exp (\mathrm{i}(k x-\omega t)) \equiv U \exp (\mathrm{i}(\xi-\bar{\omega} \tau / \eta))$. It is easy to check that the resulting dispersion relations match the first one, two or three terms of the first expansion in (8). To signify this, we will also refer to the theories (9)-(11) as $\langle 2,4\rangle,\langle 4,6\rangle$ and $\langle 6,8\rangle$ theories, respectively. The first number in angular brackets indicates the order in $\eta$ of neglected terms in the governing equation, the second indicates the order in $\eta$ of approximation error of the associated dispersion relation.

The approximation error of asymptotic theories (9)-(11) may be expressed explicitly by using the Taylor formula with the integral (Cauchy) form of the remainder term. In the case of harmonic waves the error is equal to the product of wave amplitude and approximation error of the dispersion relation.

\subsection{Non-uniqueness}

The leading order long-wave behaviour of the array is described by the hyperbolic governing equation (9) that is unable to model the dispersion produced by the micro-structure. Higher-order theories (10) and (11) do produce wave

2 Our nomenclature departs here from the more common notation where the order of asymptotic theory is equal to the order of the highest derivative. The common notation becomes inadequate when the leading order theory ceases to be hyperbolic, which will become evident during discussion of plate bending theories in Section 4.2. 
dispersion, but they also suffer from two subtly distinct yet closely related deficiencies. For example, consider the dispersion relation

$$
\bar{\omega}^{2}=\eta^{2}-\frac{1}{12} \eta^{4} \equiv \eta^{2}\left(1-\frac{1}{12} \eta^{2}\right)
$$

which is obtained by inserting $U \exp (\mathrm{i}(k x-\omega t))$ into (10) and omitting nonvanishing factors. On the one hand, it is easy to see that when $\eta>\sqrt{12}$ harmonic wave solutions become non-propagating in a sense that they do not possess either real wave number or real phase velocity. In elastodynamics this situation is usually referred to as the loss of strong ellipticity, see [6]. From the mathematical point of view, this means that the model becomes nonhypebolic, see [7]. We cannot expect a long-wave theory to be fully adequate in the short-wave regime, but the lack of hyperbolicity renders Cauchy's problem for (10) ill-posed and can make associated numerical simulations unstable. On the other hand, within the first Brillouin zone the exact dispersion relation (3) associates a unique right-travelling wave number to every frequency below cut-off. The dispersion relation (12) provides two wave numbers for each frequency, e.g. when $\bar{\omega}=0, \eta=0$ and $\eta=-\sqrt{12}$ (the negative sign is chosen to ensure the correct direction of energy propagation). The second wave number is clearly non-physical, violates asymptotic assumptions that enabled truncation of series (7), and must be considered as an artifact of the used homogenisation scheme.

The described deficiencies of (10) are, in fact, fairly typical of homogenised models. A common way to deal with the problem involves treating the leading order approximation (9) as an asymptotic identity and differentiating it twice with respect to $\xi$, so that

$$
\frac{\partial^{4} u^{*}}{\partial \xi^{2} \partial \tau^{2}} \sim \frac{\partial^{4} u^{*}}{\partial \xi^{4}}
$$

which suggests replacing the fourth order space derivative in (10) with the mixed double time double space derivative. The associated dispersion relation

$$
\bar{\omega}^{2}=\frac{\eta^{2}}{1+\eta^{2} / 12}, \quad \text { or } \quad \eta^{2}=\frac{\bar{\omega}^{2}}{1-\bar{\omega}^{2} / 12} \equiv \bar{\omega}^{2}+\frac{1}{12} \bar{\omega}^{4}+O\left(\bar{\omega}^{6}\right),
$$

indicates that the new theory has the same approximation error as (10), but is always hyperbolic and provides a unique right-propagating wave number for every frequency below cut-off. The possibility of substitution (13) is well recognised; for example, it was used in [8] for the linearised Boussinesq equation, in [9] when modelling thin plates, and recently in [10] in a homogenisation problem for long $\mathrm{SH}$ waves in a periodic bi-laminate.

The possibility of substitution (13) is a manifestation of non-uniqueness when deriving an asymptotic theory for heterogeneous structure. The advantages of theory with mixed space/time derivatives may appear obvious, nevertheless 
the choice is not quite as simple. The leading order theory (9) may as well be twice differentiated with respect to $\tau$, yielding

$$
\frac{\partial^{4} u^{*}}{\partial \tau^{4}} \sim \frac{\partial^{4} u^{*}}{\partial \xi^{2} \partial \tau^{2}}
$$

Thus, in view of (13), it is clear that the fourth order time derivative may also serve as a substitute for the fourth order space derivative in (10). The resulting dispersion relation (see the right-hand side of the identity in (14)) indicates that such theory would share all of the advantages with the theory that uses mixed derivatives. Thus, we identified three distinct approximations of equation (1), all of which reproduce the dispersion relation (3) with the same order of approximation error.

The observed non-uniqueness is related to the fact that even infinite (and convergent) asymptotic power series do not determine their sum uniquely, see [11]. Instead, they determine an equivalence class of functions with identical asymptotic expansions, which may differ by exponentially small terms. We deal with truncated series and the equivalence class of functions with identical first few terms in the asymptotic expansion is much wider. There is no simple constructive way of generating all such functions, however, it is possible to produce a useful subset of them. Indeed, if operators $\eta^{2} \partial^{2} / \partial \xi^{2}$ and $\eta^{2} \partial^{2} / \partial \tau^{2}$ are applied to (9), we obtain

$$
\frac{\partial^{4} u^{*}}{\partial \xi^{2} \partial \tau^{2}} \eta^{2}=\frac{\partial^{4} u^{*}}{\partial \xi^{4}} \eta^{2}+O\left(\eta^{4}\right) \quad \text { and } \quad \frac{\partial^{4} u^{*}}{\partial \tau^{4}} \eta^{2}=\frac{\partial^{4} u^{*}}{\partial \xi^{2} \partial \tau^{2}} \eta^{2}+O\left(\eta^{4}\right)
$$

respectively. Since the approximation errors of (10) and (16) are identical, superposition (10) $-C_{1} \times(16)_{1}-C_{2} \times(16)_{2}$ would also be a fourth order longwave model for our lattice structure. We may write it explicitly as

$$
\begin{aligned}
{\left[1-\eta^{2}\left(C_{1}-C_{2}\right) \frac{\partial^{2}}{\partial \xi^{2}}-\eta^{2} C_{2} \frac{\partial^{2}}{\partial \tau^{2}}\right] \frac{\partial^{2} u^{*}}{\partial \tau^{2}}=} & \\
& {\left[1+\eta^{2}\left(\frac{1}{12}-C_{1}\right) \frac{\partial^{2}}{\partial \xi^{2}}\right] \frac{\partial^{2} u^{*}}{\partial \xi^{2}}+O\left(\eta^{4}\right), }
\end{aligned}
$$

where $C_{1}$ and $C_{2}$ are arbitrary constants. The derivation of (17) involves differentiation of asymptotic series and therefore is purely formal. However, the assumptions that enabled truncation of the infinite series in (7) ensure that this new theory has the same asymptotic accuracy as the original theory (10).

Previously, there have been other attempts to derive general classes of longwave theories, similar to (17). In particular, Metrikine \& Askes [12] explicitly introduced a non-locality into their definition of the displacement field; so modified homogenisation procedure enabled them to produce one-parameter classes of fourth and sixth order models. Asymptotic theory (17) is formally 
identical to the fourth order model from [12] when $C_{2}=0$. Metrikine developed these ideas further in [13], where he argued in favour of theories with higher inertia gradients using a range of phenomenological considerations, formal comparisons with the Timoshenko and Mindlin beam and rod theories, as well as an explicit derivation of a relevant model for concrete.

\subsection{Parameter selection}

In order to formulate the criteria for selecting parameters $C_{1}$ and $C_{2}$ it is necessary to study the dispersion relation of the theory (17), given by

$$
\bar{\omega}^{2}+\left(C_{1}-C_{2}\right) \bar{\omega}^{2} \eta^{2}+C_{2} \bar{\omega}^{4}=\eta^{2}-\left(\frac{1}{12}-C_{1}\right) \eta^{4}
$$

Since equation (18) is quadratic both in $\eta^{2}$ and in $\bar{\omega}^{2}$, it associates two (not necessarily real) frequencies with every wave number and two (possibly, imaginary) wave numbers with every frequency. Our original mechanical model does not exhibit such a complex behaviour, associated with higher gradients of strain and inertia entering the governing equation. This may be interpreted as introduction of both spatial and temporal non-locality into the model. Another useful way of understanding the situation would be to assume that we are trying to reproduce the behaviour of the original single atom chain, in which interactions happen between nearest neighbours only, using a more complex lattice structure. Non-single-valuedness in $\eta^{2}$ corresponds to structures where atoms simultaneously interact with multiple neighbours, whereas non-singlevaluedness in $\bar{\omega}^{2}$ corresponds to lattices comprising multiple types of atoms, see $[14]$.

Both inertia and strain gradients are capable of describing the dispersion of waves in the lattice structure, however both of them induce spurious features into the response. Governing equations with higher gradients of strain may have solutions with spurious non-physical terms, associated with extraneous solutions of the dispersion relation, as in (12). For instance, the low-frequency expansion of the extraneous solution in (18) is given by

$$
\eta^{2}=\frac{12}{1-12 C_{1}}-\frac{1-12 C_{2}}{1-12 C_{1}} \bar{\omega}^{2}+O\left(\bar{\omega}^{4}\right)
$$

so its limit becomes imaginary when $C_{1}>1 / 12$ and does not exist when $C_{1}=1 / 12$. A real low-frequency limit of (19) corresponds to the loss of hyperbolicity, thus we term the associated theories numerically unstable. Higher inertia gradient terms result in the presence of extraneous high-frequency branches in the dispersion relation. In particular, the long-wave expansion 
of the extraneous branch in (18) may be written as

$$
\bar{\omega}^{2}=-\frac{1}{C_{2}}-\frac{C_{1}}{C_{2}} \eta^{2}+O\left(\eta^{4}\right)
$$

thus $C_{2}<0$ corresponds to the presence of spurious propagating solutions for frequencies $\bar{\omega}>\sqrt{-1 / C_{2}}$. Consequently, we cannot use (17) above the extraneous branch cut-off $\bar{\omega}=\sqrt{-1 / C_{2}}$ and will refer to this situation by saying that the theory (17) is bandwidth-limited when $C_{2}<0$. While the numerically unstable theories are not suitable for numerical simulations, bandwidth-limited theories may describe both stationary and non-stationary dynamics of microstructure at frequencies below the extraneous branch cut-off. In fact, many relevant engineering problems are already bandwidth-limited, for example vibration of thin plates or stress pulse propagation in thin bars.

A more subtle difference between the theories with strain and inertia gradients is related to the choice of appropriate boundary and initial conditions. In order to use the theory with higher strain gradients and, therefore, spatial nonlocality manifested in the presence of extraneous solutions, it is necessary to formulate additional boundary conditions. The difficulty of formulating consistent conditions may be greater than the derivation of the asymptotic theory itself. On the contrary, theories with inertia gradients require additional initial conditions, which are only necessary for non-stationary problems. Thus, it appears to be beneficial to consider theories that contain higher gradients of inertia only. Generally, this can only be achieved in a scalar context and was previously done in [9] for asymptotic models of thin plates. The resulting theories were termed "dynamic" to signify their likely applications. Kaplunov, Kossovich \& Nolde [4] refer to these asymptotic theories as theories with modified inertia. This is the term that we will use in our paper.

The class of theories with modified inertia may be obtained from (17) by specifying $C_{1}=1 / 12$. The associated dispersion relation is obtained from (18) by solving for $\eta^{2}$, expanding for small $\bar{\omega}$, and yielding

$$
\eta^{2}=\frac{\bar{\omega}^{2}\left(1+C_{2} \bar{\omega}^{2}\right)}{1+\left(C_{2}-1 / 12\right) \bar{\omega}^{2}} \equiv \bar{\omega}^{2}+\frac{\bar{\omega}^{4}}{12}+\left(\frac{1}{144}-\frac{C_{2}}{12}\right) \bar{\omega}^{6}+O\left(\bar{\omega}^{8}\right) .
$$

Parameter $C_{2}$ may be chosen arbitrarily, e.g. by numerical fitting of the approximate dispersion relation (21) into the exact dispersion relation (3). For example, the theory with $C_{2}=-0.0635$ reproduces the exact dispersion relation for $\bar{\omega}<1.62$ with $0.1 \%$ relative accuracy. The theory with $C_{2}=-0.0804$ is $1 \%$ accurate for $\bar{\omega}<1.93$.

Probably the best analytic guess for value of $C_{2}$ may be obtained by matching the third order term of the expansion in (21) with the appropriate term of (8), thus $C_{2}=-1 / 20$. Firstly and obviously, this ensures that the governing equa- 


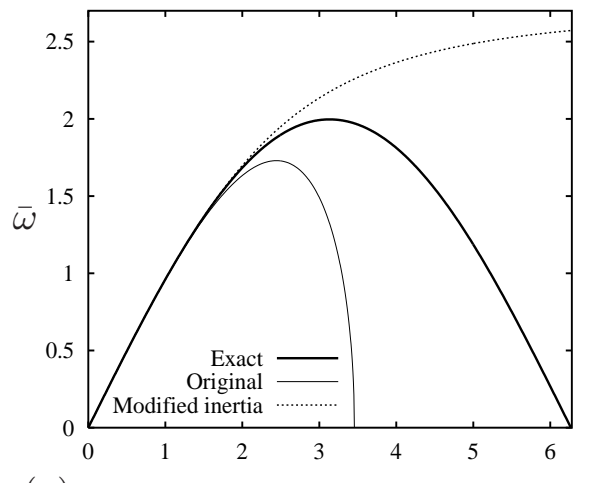

(a)

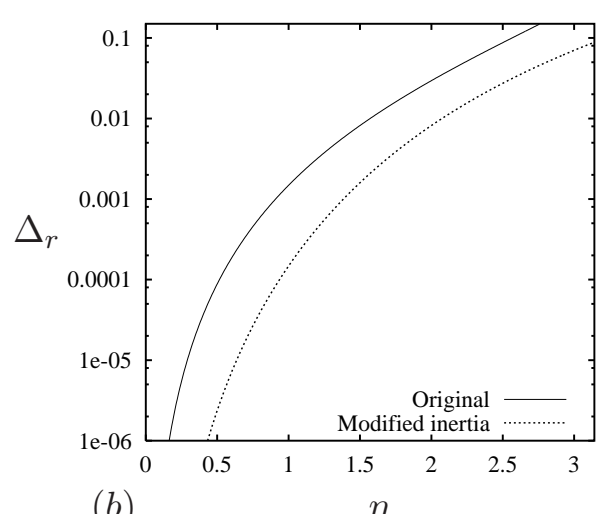

(b)

Fig. 2. Exact (thick solid) and approximate (thin solid and dotted) dispersion curves for the regular array of particles: (a) frequency of harmonic waves against wave number; (b) relative approximation error against wave number.

tion of our asymptotic theory is accurate to $O\left(\eta^{8}\right)$. Secondly, the dispersion relation (21) may be recognised as a [1/1] Padé approximant in $\bar{\omega}^{2}$ for the exact dispersion relation (3), see [15]. The dispersion relation of the proposed asymptotic theory would share both the improved asymptotic accuracy and good numerical convergence properties of the Padé approximant. This links our model to the one described in [16], however, their approach does not generate higher gradients of inertia. It is worth remarking that the dispersion relation (18) may also be interpreted as a quadratic Padé approximant, see [3]. Higher order theories would produce higher order generalised Padé (or Hermite-Padé) approximants.

The resulting $\langle 4,8\rangle$ theory with modified inertia $\left(C_{1}=1 / 12\right.$ and $\left.C_{2}=-1 / 20\right)$ has the following dimensional representation

$$
\left(1-\frac{2 l^{2}}{15} \frac{\partial^{2}}{\partial x^{2}}+\frac{1}{20 \Omega^{2}} \frac{\partial^{2}}{\partial t^{2}}\right) \ddot{u}=\Omega^{2} \frac{\partial^{2} u}{\partial x^{2}} l^{2} .
$$

Its numerical performance is demonstrated in Figure 2. Figure 2(a) presents the dispersion curves for the exact dispersion relation (3), as well as for original (10) and modified inertia (22) theories. Better accuracy of (22) in the medium wavelength range is apparent by inspection. The plot of relative approximation errors in Figure 2(b) indicates that the new theory offers at least half an order reduction of error magnitude when compared to (10).

\subsection{Application to statics}

The parameter selection criteria indicated in the previous section may at first appear confusing. While the reasons for using theories with inertia gradients are fairly convincing when applied to wave propagation problems, they may 
appear less appealing in statics. Indeed, our favoured theory (22), when specialised to statics, does not possess any higher-gradient terms at all!

This behaviour is not coincidental. When fine-tuning asymptotic theories for problems of wave propagation we had full information about characteristic lengths of the involved wave fields. On the contrary, we have not specified a characteristic length scale of the static problem of interest and therefore cannot rationally discriminate between various theories. Thus, to formulate a statics theory we need to reformulate governing equation (5) by introducing an external force field $\mathcal{F}=\mathcal{F}(x)$ and yielding

$$
\sum_{n=1}^{\infty} \frac{2}{(2 n) !} \frac{\partial^{2 n} u}{\partial x^{2 n}} l^{2 n}+\frac{\mathcal{F}}{K}=0
$$

If we now require that $\mathcal{F}$ is sufficiently smooth and periodic with the period $L \gg l$, equation (23) may again be non-dimensionalised according to (6) with $\eta=l / L$ and truncated. An appropriate fourth order theory would take the following form

$$
\frac{\partial^{2} u^{*}}{\partial \xi^{2}}+\frac{l \mathcal{F}}{K}+\frac{1}{12} \frac{\partial^{4} u^{*}}{\partial \xi^{4}} \eta^{2}=O\left(\eta^{4}\right),
$$

where $u^{*}(\xi) \equiv \eta^{2} l u(x(\xi))$. By considering the leading order terms of this equation it is clear that the higher strain gradient term is not arbitrary, but depends on the gradient of external stress. The appropriate class of asymptotically equivalent theories may then be obtained by twice differentiating the leading order of (24) and superimposing it with the original theory, so that

$$
\frac{\partial^{2} u^{*}}{\partial \xi^{2}}+\frac{l \mathcal{F}}{K}+\left(\left(\frac{1}{12}-C\right) \frac{\partial^{4} u^{*}}{\partial \xi^{4}}-\frac{C l}{K} \frac{\partial^{2} \mathcal{F}}{\partial \xi^{2}}\right) \eta^{2}=O\left(\eta^{4}\right)
$$

in which $C$ is an arbitrary constant. A glance at (25) reveals that our previous statement that $\mathcal{F}$ has to be sufficiently smooth should be interpreted as the requirement that $\partial^{2} \mathcal{F} / \partial \xi^{2}$ is of the same asymptotic order as $\mathcal{F}$.

\subsection{Theories of order six and higher}

The formal procedure employed in the previous sections may be naturally extended to higher-order asymptotic theories, however the benefits of such generalisation are ambiguous. First, the numerical accuracy of the asymptotic model is typically maximised when it is truncated at the optimal (numerically smallest) term, see e.g. [17]. Second, difficulties with numerical implementation of higher-order derivatives may overweight the benefits of using asymptotic model. Thus, we only provide indicative examples of higher-order theories in this paper. 
The class of sixth-order theories, asymptotically equivalent to the theory (11), may be generated by applying operators $\eta^{4} \partial^{4} / \partial \xi^{4}, \eta^{4} \partial^{4} / \partial \xi^{2} \partial \tau^{2}$ and $\eta^{4} \partial^{4} / \partial \tau^{4}$ to asymptotic equality (9) and $\eta^{2} \partial^{2} / \partial \xi^{2}$ and $\eta^{2} \partial^{2} / \partial \tau^{2}$ to (10). The superposition of the resulting asymptotic equalities may be written as

$$
\begin{aligned}
& {\left[1-\eta^{2}\left(C_{1}-C_{2}\right) \frac{\partial^{2}}{\partial \xi^{2}}-\eta^{2} C_{2} \frac{\partial^{2}}{\partial \tau^{2}}+\eta^{4}\left(C_{3}-C_{4}+\frac{C_{2}}{12}\right) \frac{\partial^{4}}{\partial \xi^{4}}\right.} \\
& \left.\quad+\eta^{4}\left(C_{4}-C_{5}\right) \frac{\partial^{4}}{\partial \xi^{2} \partial \tau^{2}}+\eta^{4} C_{5} \frac{\partial^{4}}{\partial \tau^{4}}\right] \frac{\partial^{2} u^{*}}{\partial \tau^{2}}= \\
& {\left[1+\eta^{2}\left(\frac{1}{12}-C_{1}\right) \frac{\partial^{2}}{\partial \xi^{2}}+\eta^{4}\left(\frac{1}{360}-\frac{C_{1}}{12}+C_{3}\right) \frac{\partial^{4}}{\partial \xi^{4}}\right] \frac{\partial^{2} u^{*}}{\partial \xi^{2}}+O\left(\eta^{6}\right),}
\end{aligned}
$$

where $C_{1}, \ldots, C_{5}$ are arbitrary constants. The class of theories obtained in [12] is reproduced when $C_{2}=C_{4}=C_{5}=0$ and $C_{3}$ is a certain function of $C_{1}$. Theories (26) are numerically stable when

$$
6 D \equiv 6 C_{1}^{2}+C_{1}-24 C_{3}-\frac{1}{40} \leqslant 0 \quad \text { or } \quad \frac{12 C_{1}-1+2 \sqrt{D}}{2 C_{1}-24 C_{3}-1 / 15} \leqslant 0 .
$$

Theories with modified inertia are obtained by selecting $C_{1}=1 / 12, C_{3}=$ $1 / 240$ and $240 C_{4}=20 C_{2}+1$, parameters $C_{2}$ and $C_{5}$ may be chosen arbitrarily. In particular, when $C_{2}=-49 / 276$ and $C_{5}=79 / 28980$ the dispersion relation of the theory (26) is a [2/2] Padé approximant in $\bar{\omega}^{2}$ of the exact dispersion relation for $\bar{v}^{2}$. However, the numerical performance of so-formulated $\langle 6,12\rangle$ theory is not significantly better and, for higher wave numbers, worse than the corresponding $\langle 4,8\rangle$ theory $(22)$.

\section{Two-dimensional square lattice structure}

Examples in the previous section demonstrated how gradients of strain may be adjoined or replaced by gradients of inertia in one-dimensional problems and the advantages of so-formulated models. The technique is easily generalised to those two-dimensional problems that are still scalar in nature, i.e. that are reducible to a problem for a combination of uncoupled scalar potentials. For example, this is the case for isotropic media as well as certain special types of anisotropy, see [18]. In order to illustrate the difficulties one encounters when dealing with non-scalar models we consider a somewhat simplified version of a square lattice structure studied previously in [19], see Figure 3. The equations 


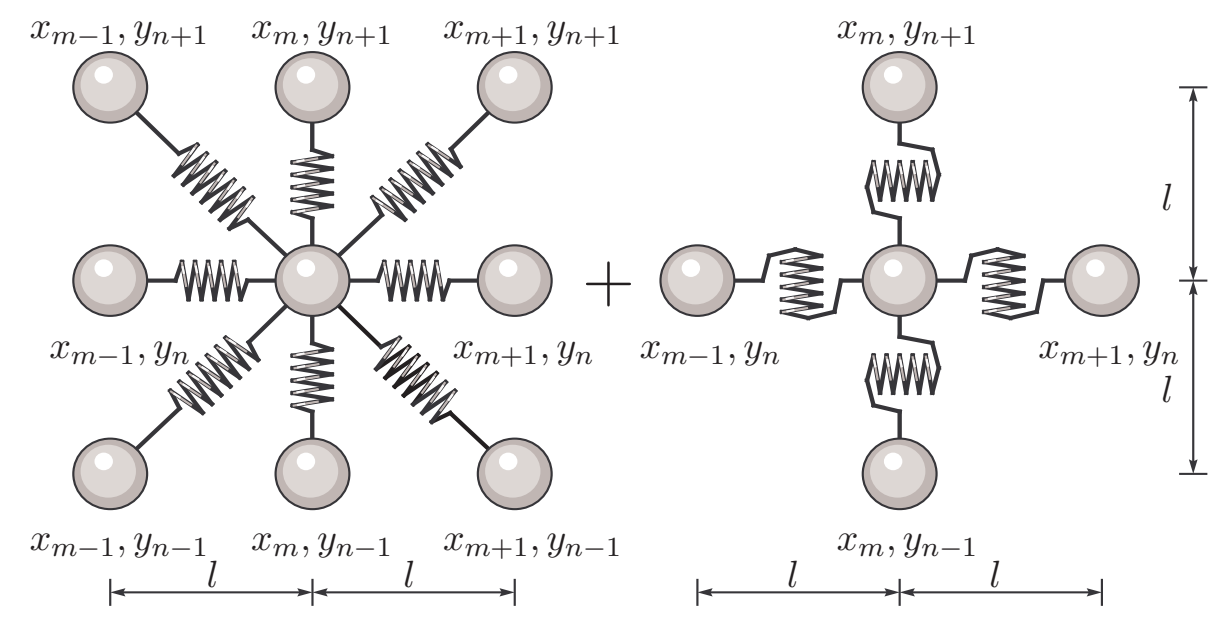

Fig. 3. Two-dimensional square lattice structure.

of motion for the structure are given by

$$
\begin{aligned}
& M \ddot{u}_{m, n}= K_{1}\left(u_{m, n-1}-2 u_{m, n}+u_{m, n+1}\right)+K_{2}\left(u_{m-1, n}-2 u_{m, n}+u_{m+1, n}\right) \\
&+ K_{0}\left(u_{m-1, n-1}+u_{m+1, n-1}+u_{m-1, n+1}+u_{m+1, n+1}+v_{m-1, n-1}\right. \\
&\left.-v_{m+1, n-1}-v_{m-1, n+1}+v_{m+1, n+1}-4 u_{m, n}\right) / 2 \\
& M \ddot{v}_{m, n}=K_{1}\left(v_{m-1, n}-2 v_{m, n}+v_{m+1, n}\right)+K_{2}\left(v_{m, n-1}-2 v_{m, n}+v_{m, n+1}\right) \\
&+K_{0}\left(v_{m-1, n-1}+v_{m-1, n+1}+v_{m+1, n-1}+v_{m+1, n+1}+u_{m-1, n-1}\right. \\
&\left.-u_{m-1, n+1}-u_{m+1, n-1}+u_{m+1, n+1}-4 v_{m, n}\right) / 2
\end{aligned}
$$

where $(u, v)_{m, n}$ is the displacement vector for a particle situated at $\left(x_{m}, y_{n}\right) \equiv$ $(m l, n l), m, n \in \mathbb{I}$, with $l$ denoting interparticle distance. $M$ is the particle mass, and $K_{0}, K_{1}$, and $K_{2}$ are the stiffnesses of diagonal longitudinal, axial longitudinal and axial shear springs, respectively.

The dynamic behaviour of the lattice may be assessed by considering propagation of harmonic waves, defined as

$$
(u, v)_{m, n}=(U, V) e^{\mathrm{i}\left(k c_{\theta} x_{m}+k s_{\theta} y_{n}-\omega t\right)},
$$

where $t$ is time, $k$ is wave number and $\omega$ is circular frequency, as before. Parameters $c_{\theta}$ and $s_{\theta}$ are direction cosines for the wave normal, $c_{\theta}^{2}+s_{\theta}^{2}=$ 1. By inserting solutions (30) into equations of motion (28)-(29), the exact dispersion relation for the square lattice is obtained in the form

$$
\begin{aligned}
& \sin ^{2}\left(\eta c_{\theta}\right) \sin ^{2}\left(\eta s_{\theta}\right)= \\
& \quad\left(\bar{\omega}^{2}+\beta_{1} \cos \left(\eta s_{\theta}\right)+\cos \left(\eta c_{\theta}\right) \cos \left(\eta s_{\theta}\right)+\beta_{2} \cos \left(\eta c_{\theta}\right)-\beta_{1}-\beta_{2}-1\right) \\
& \quad \times\left(\bar{\omega}^{2}+\beta_{1} \cos \left(\eta c_{\theta}\right)+\cos \left(\eta c_{\theta}\right) \cos \left(\eta s_{\theta}\right)+\beta_{2} \cos \left(\eta s_{\theta}\right)-\beta_{1}-\beta_{2}-1\right),
\end{aligned}
$$

within which we introduced

$$
\beta_{1}=\frac{K_{1}}{K_{0}}, \quad \beta_{2}=\frac{K_{2}}{K_{0}}, \quad \bar{\omega}=\sqrt{\frac{M}{2 K_{0}}} \omega, \quad \text { and } \quad \eta=k l .
$$


Numerical analysis of (31) reveals the presence of two long-wave low-frequency solution branches that are coupled because of the lattice structure anisotropy and may be referred to as quasi-longitudinal (QL) and quasi-transversal (QT). The long-wave limits of the phase velocity $\bar{v}=\sqrt{2} \bar{\omega} / \eta$ for these modes are the solutions of the secular equation

$$
\bar{v}_{0}^{4}-(\alpha+\gamma) \bar{v}_{0}^{2}+\alpha \gamma s_{\theta}^{4}-\left(4-\alpha^{2}-\gamma^{2}\right) s_{\theta}^{2} c_{\theta}^{2}+\alpha \gamma c_{\theta}^{4}=0, \quad \eta \rightarrow 0,
$$

where $\alpha \equiv \beta_{1}+1$ and $\gamma \equiv \beta_{2}+1$. A more general ansatz $\bar{v}^{2}=\bar{v}_{0}^{2}+\bar{v}_{2}^{2} \eta^{2}+\bar{v}_{4}^{2} \eta^{4}+$ $O\left(\eta^{6}\right)$ may be used in conjunction with (31) to determine further refinements to the leading order long-wave approximation (33), but the amount of algebra involved into such analysis cannot be justified within this paper. We will, therefore, refine our asymptotic theories on the basis of long-wave expansions of (31) along particular directions $\left(c_{\theta}, s_{\theta}\right) \equiv(\cos \theta, \sin \theta)$, where $\theta=0, \theta=\pi / 4$ or $\theta=\pi / 2$. Longitudinal and transversal modes are not coupled along these directions and the appropriate expansions for both long-wave branches are given explicitly as

$$
\begin{gathered}
\frac{\bar{v}_{L}^{2}}{\alpha}=\frac{\bar{v}_{T}^{2}}{\gamma}=1-\frac{\eta^{2}}{12}+\frac{\eta^{4}}{360}+O\left(\eta^{6}\right), \quad \theta=0 \quad \text { or } \frac{\pi}{2}, \\
\frac{2 \bar{v}_{L}^{2}}{\alpha+\gamma+2}=1-\frac{(\alpha+\gamma+14)}{24(\alpha+\gamma+2)} \eta^{2}+\frac{(\alpha+\gamma+62)}{1440(\alpha+\gamma+2)} \eta^{4}+O\left(\eta^{6}\right), \\
\frac{2 \bar{v}_{T}^{2}}{\alpha+\gamma-2}=1-\frac{\eta^{2}}{24}+\frac{\eta^{4}}{1440}+O\left(\eta^{6}\right), \quad \theta=\frac{\pi}{4} .
\end{gathered}
$$

\subsection{Long-wave models}

We employ virtually identical homogenisation procedure to the one used for one-dimensional array of particles. Specifically, we non-dimensionalise equations of motion (28)-(29) according to

$$
x=\xi / k, \quad y=\zeta / k, \quad t=\tau \sqrt{M} /\left(\eta \sqrt{K_{0}}\right),
$$

and expand the displacements into the Taylor series around a central particle $(u, v) \equiv(u, v)_{m, n}$. After appropriately truncating the series, we obtain a $\langle 4,6\rangle$ 
asymptotic theory governed by the following equations of motion

$$
\begin{aligned}
\gamma \frac{\partial^{2} u}{\partial \xi^{2}} & +2 \frac{\partial^{2} v}{\partial \xi \partial \zeta}+\alpha \frac{\partial^{2} u}{\partial \zeta^{2}}-\frac{\partial^{2} u}{\partial \tau^{2}} \\
& +\frac{1}{3}\left(\frac{\gamma}{4} \frac{\partial^{4} u}{\partial \xi^{4}}+\frac{\partial^{4} v}{\partial \xi^{3} \partial \zeta}+\frac{3}{2} \frac{\partial^{4} u}{\partial \xi^{2} \partial \zeta^{2}}+\frac{\partial^{4} v}{\partial \xi \partial \zeta^{3}}+\frac{\alpha}{4} \frac{\partial^{4} u}{\partial \zeta^{4}}\right) \eta^{2}=O\left(\eta^{4}\right) \\
\alpha \frac{\partial^{2} v}{\partial \xi^{2}} & +2 \frac{\partial^{2} u}{\partial \xi \partial \zeta}+\gamma \frac{\partial^{2} v}{\partial \zeta^{2}}-\frac{\partial^{2} v}{\partial \tau^{2}} \\
& +\frac{1}{3}\left(\frac{\alpha}{4} \frac{\partial^{4} v}{\partial \xi^{4}}+\frac{\partial^{4} u}{\partial \xi^{3} \partial \zeta}+\frac{3}{2} \frac{\partial^{4} v}{\partial \xi^{2} \partial \zeta^{2}}+\frac{\partial^{4} u}{\partial \xi \partial \zeta^{3}}+\frac{\gamma}{4} \frac{\partial^{4} v}{\partial \zeta^{4}}\right) \eta^{2}=O\left(\eta^{4}\right)
\end{aligned}
$$

The essentially non-scalar nature of the coupled equations (37)-(38) is manifested by the presence of a number of additional gradient terms, when compared to e.g. (10) or (11). This results in a higher number of options when equations (37)-(38) are generalised. To this end, we consider superpositions of (37) with $\eta^{2} \partial^{2} / \partial \xi^{2}, \eta^{2} \partial^{2} / \partial \zeta^{2}$ and $\eta^{2} \partial^{2} / \partial \tau^{2}$ applied to (37) and $\eta^{2} \partial^{2} / \partial \xi \partial \zeta$ applied to (38), i.e. $\left(1+\eta^{2}\left(C_{11} \partial^{2} / \partial \xi^{2}+C_{12} \partial^{2} / \partial \zeta^{2}+C_{13} \partial^{2} / \partial \tau^{2}\right)\right) \times(37)+C_{14} \times(38)$. This yields

$$
\begin{aligned}
\gamma \frac{\partial^{2} u}{\partial \xi^{2}} & +2 \frac{\partial^{2} v}{\partial \xi \partial \zeta}+\alpha \frac{\partial^{2} u}{\partial \zeta^{2}}-\frac{\partial^{2} u}{\partial \tau^{2}}+\left[\gamma\left(C_{11}+\frac{1}{12}\right) \frac{\partial^{4} u}{\partial \xi^{4}}+\alpha\left(C_{12}+\frac{1}{12}\right) \frac{\partial^{4} u}{\partial \zeta^{4}}\right. \\
& +\left(2 C_{11}+\alpha C_{14}+\frac{1}{3}\right) \frac{\partial^{4} v}{\partial \xi^{3} \partial \zeta}+\left(\alpha C_{11}+\gamma C_{12}+2 C_{14}+\frac{1}{2}\right) \frac{\partial^{4} u}{\partial \xi^{2} \partial \zeta^{2}} \\
& +\left(2 C_{12}+\gamma C_{14}+\frac{1}{3}\right) \frac{\partial^{4} v}{\partial \xi \partial \zeta^{3}}+\left(2 C_{13}-C_{14}\right) \frac{\partial^{4} v}{\partial \tau^{2} \partial \xi \partial \zeta}-C_{13} \frac{\partial^{4} u}{\partial \tau^{4}} \\
& \left.+\left(\gamma C_{13}-C_{11}\right) \frac{\partial^{4} u}{\partial \tau^{2} \partial \xi^{2}}+\left(\alpha C_{13}-C_{12}\right) \frac{\partial^{4} u}{\partial \tau^{2} \partial \zeta^{2}}\right] \eta^{2}=O\left(\eta^{4}\right)
\end{aligned}
$$

where $C_{11}, C_{12}, C_{13}$ and $C_{14}$ are arbitrary constants. The second generalised governing equation is obtained in a similar fashion, by combining (38) with $\eta^{2} \partial^{2} / \partial \xi^{2}, \eta^{2} \partial^{2} / \partial \zeta^{2}$ and $\eta^{2} \partial^{2} / \partial \tau^{2}$ applied to (38) and $\eta^{2} \partial^{2} / \partial \xi \partial \zeta$ applied to (37), with the result $\left(1+\eta^{2}\left(C_{21} \partial^{2} / \partial \xi^{2}+C_{22} \partial^{2} / \partial \zeta^{2}+C_{23} \partial^{2} / \partial \tau^{2}\right)\right) \times(38)$ $+C_{24} \times(37)$ given by

$$
\begin{aligned}
\alpha \frac{\partial^{2} v}{\partial \xi^{2}} & +2 \frac{\partial^{2} u}{\partial \xi \partial \zeta}+\gamma \frac{\partial^{2} v}{\partial \zeta^{2}}-\frac{\partial^{2} v}{\partial \tau^{2}}+\left[\alpha\left(C_{21}+\frac{1}{12}\right) \frac{\partial^{4} v}{\partial \xi^{4}}+\gamma\left(C_{22}+\frac{1}{12}\right) \frac{\partial^{4} v}{\partial \zeta^{4}}\right. \\
& +\left(2 C_{21}+\gamma C_{24}+\frac{1}{3}\right) \frac{\partial^{4} u}{\partial \xi^{3} \partial \zeta}+\left(\gamma C_{21}+\alpha C_{22}+2 C_{24}+\frac{1}{2}\right) \frac{\partial^{4} v}{\partial \xi^{2} \partial \zeta^{2}} \\
& +\left(2 C_{22}+\alpha C_{24}+\frac{1}{3}\right) \frac{\partial^{4} u}{\partial \xi \partial \zeta^{3}}+\left(2 C_{23}-C_{24}\right) \frac{\partial^{4} u}{\partial \tau^{2} \partial \xi \partial \zeta}-C_{23} \frac{\partial^{4} v}{\partial \tau^{4}} \\
& \left.+\left(\alpha C_{23}-C_{21}\right) \frac{\partial^{4} v}{\partial \tau^{2} \partial \xi^{2}}+\left(\gamma C_{23}-C_{22}\right) \frac{\partial^{4} v}{\partial \tau^{2} \partial \zeta^{2}}\right] \eta^{2}=O\left(\eta^{4}\right)
\end{aligned}
$$

Again, constants $C_{21}, C_{22}, C_{23}$ and $C_{24}$ may be chosen arbitrarily. 
An inspection of generalised governing equations (39)-(40) immediately reveals that they have ten different fourth order spatial derivative terms. Since a theory with modified inertia cannot possess higher order spatial derivatives, it would require vanishing of all ten corresponding coefficients in both governing equations. Unfortunately, these coefficients depend on only eight arbitrary constants. Thus, it is generally impossible to select $C_{11}, \ldots, C_{24}$ such that all of the higher order spatial derivative terms vanish from (39)-(40), except, possibly, for some special combinations of spring stiffnesses. This also suggests that in the non-scalar context, generally, it is not possible to create asymptotic theories that do not possess extraneous solutions. Therefore, spatial non-locality is an unavoidable feature of vector asymptotic models and if the model is intended for using on finite domains, the appropriate boundary conditions must be formulated.

\subsection{Example of parameter selection}

Detailed analysis of the options available for selecting parameters for (39)-(40) is a fairly complex and, to a large extent, numerical problem. Thus, we only indicate a possibly useful parameter selection procedure, with no claims at generality or optimality. We start by noticing that in order for the asymptotic theory to reproduce the expected symmetric response for propagation along $O \xi$ and $O \zeta$, it is necessary to set $C_{21}=C_{12}, C_{22}=C_{11}, C_{23}=C_{13}$ and $C_{24}=C_{14}$. The numerical stability of (39)-(40) can be ensured by using necessary and sufficient conditions for stability when propagating along $\theta=0$ and $\theta=\pi / 2$, which are given by

$$
C_{11} \leqslant-\frac{1}{12}, \quad C_{12} \leqslant-\frac{1}{12}
$$

We also use necessary and sufficient condition for stability when propagating along $\theta=\pi / 4$ that is equivalent to ensuring that the following bi-quadratic equation for $\eta$ has no real roots

$$
\left(C^{\prime}-C_{14}\right)\left(1+\mu\left(C^{\prime}+C_{14}\right)\right) \eta^{4}-12\left(1+2 \mu C^{\prime}\right) \eta^{2}+24 \mu=0,
$$

where $C^{\prime}=1 / 12+C_{11}+C_{12}$ and $\mu=2+\alpha+\gamma$. Since conditions (41)-(42) are not sufficient to ensure numerical stability for all $\theta$, additional numerical tests have to be performed to ensure the numerical stability for all directions of propagation.

It is impossible to refine the asymptotic accuracy of the model (39)-(40) for all of $\theta \in\{0, \pi / 4, \pi / 2\}$. For our specific choice of parameters $\alpha=6 / 5$ and $\gamma=9 / 10$, it turned out beneficial to match $O\left(\eta^{4}\right)$ terms in the expansions (34) 


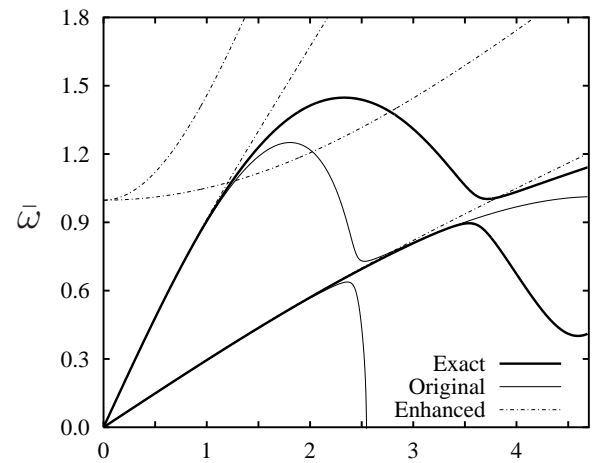

(a)

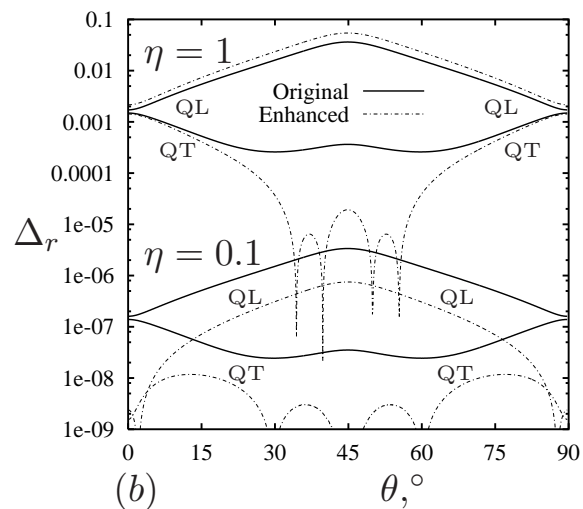

(b)

Fig. 4. Exact (thick solid) and approximate (thin solid, dotted and dashed) dispersion curves for asymptotic models of the 2D square lattice structure: (a) frequency of harmonic waves against wave number when $\theta=\pi / 6$; (b) relative approximation error against the direction of propagation.

by selecting

$$
C_{11}=-\frac{\alpha-\gamma\left(30 C_{12}+1\right)}{30 \alpha}, \quad C_{13}=-\frac{30 C_{12}+1}{30 \alpha} .
$$

Only one of the expansions (35) can be further refined; we chosen $C_{13}$ such that the resulting theory is less limited in bandwidth. Finally, the value of $C_{12}$ was chosen by considering numerical stability conditions (41)-(42), with the resulting set of parameters given by

$$
\begin{gathered}
C_{11}=C_{22}=-\frac{641}{1320}, \quad C_{12}=C_{21}=-\frac{7}{11} \\
C_{13}=C_{23}=\frac{199}{396}, \quad C_{14}=C_{24}=-\frac{514}{495}
\end{gathered}
$$

The numerical performance of so defined model is illustrated in Figure 4. The dispersion curves for frequency versus wave number are given in Figure 4(a) for $\theta=\pi / 6$. There are two speeds of sound in this vector problem and two exact dispersion curves (thick solid) exhibit strong coupling behaviour for $\eta \approx$ 3.5. The original theory (37)-(38) (thin solid curves) crudely reproduces this coupling behaviour. The numerical instability of the theory manifests itself by the first branch becoming non-propagating at $\eta \approx 2.5$. The refined asymptotic theory (39)-(40), (44) (dotted and dashed curves) has two spurious branches with cut-off frequency $\bar{\omega} \approx 1$ and, therefore, is bandwidth-limited. However, both relevant (long-wave low-frequency) branches are numerically stable; this seems to be true for all $\theta$. The accuracy can be assessed by considering relative approximation errors plotted in Figure 4(b) for two fixed values of $\eta$ and a full range of angles $\theta$. 


\section{Application to asymptotic structural theories}

Although the term "strain gradient theory" is usually associated with the modelling of micro-structure, long-wave approximations for simple engineering structures — rods, beams, plates and shells - were among the first asymptotic theories with higher gradients of strain to be derived and used in practice. Many modern gradient models of micro-structure are governed by the equations that are formally equivalent to asymptotic models of simple structures. We illustrate this similarity by using our procedure in the context of several well-known structural theories.

\subsection{Longitudinal waves in a cylindrical rod}

One of the first structural theories to incorporate higher gradients of strain was the theory for longitudinal waves in a thin cylindrical rod of radius $R$. The higher order (lateral inertia) correction was proposed by Rayleigh [20]; Love [21] derived the appropriate governing equation

$$
c_{0}^{2} \frac{\partial^{2} u}{\partial x^{2}}=\left(1-\frac{\nu^{2} R^{2}}{2} \frac{\partial^{2}}{\partial x^{2}}\right) \ddot{u},
$$

where $u=u(x, t)$ is the longitudinal displacement, $\nu$ is the Poisson ratio, $c_{0} \equiv$ $\sqrt{E / \rho}$ the bar velocity, $E$ the Young modulus and $\rho$ the material density. The dispersion relation of (45) matches two terms of the long-wave low-frequency expansion of the Pochhammer-Chree dispersion relation, see e.g. [22], which may be written as

$$
\eta^{2}=\bar{\omega}^{2}+\frac{\nu^{2}}{2} \bar{\omega}^{4}+\frac{\nu^{2}\left(4 \nu^{3}-8 \nu^{2}-4 \nu+7\right)}{48\left(1-\nu^{2}\right)} \bar{\omega}^{6}+O\left(\bar{\omega}^{8}\right),
$$

with $\bar{\omega} \equiv \omega R / c_{0}$ and $\eta=k R$. Therefore, Love's equation (45) is a $\langle 4,6\rangle$ asymptotic theory for cylindrical rods.

Governing equation (45) may be recognised as a particular case of the fourth order theory for one-dimensional array of particles (17). Thus, we introduce similar scaling $\xi=k x, \tau=c_{0} k t$, and generate a class of theories in the form

$$
\left(1-\eta^{2} C_{1} \frac{\partial}{\partial \xi^{2}}\right) \frac{\partial^{2} u^{*}}{\partial \xi^{2}}=\left(1-\eta^{2} C_{2} \frac{\partial}{\partial \tau^{2}}-\eta^{2}\left(C_{1}-C_{2}+\frac{\nu^{2}}{2}\right) \frac{\partial}{\partial \xi^{2}}\right) \frac{\partial^{2} u^{*}}{\partial \tau^{2}}
$$

in which $u^{*} \equiv l u$. The associated dispersion relation is given by

$$
C_{2} \bar{\omega}^{4}+\bar{\omega}^{2}+\left(\frac{\nu^{2}}{2}+C_{1}-C_{2}\right) \bar{\omega}^{2} \eta^{2}-\eta^{2}-C_{1} \eta^{4}=0 .
$$




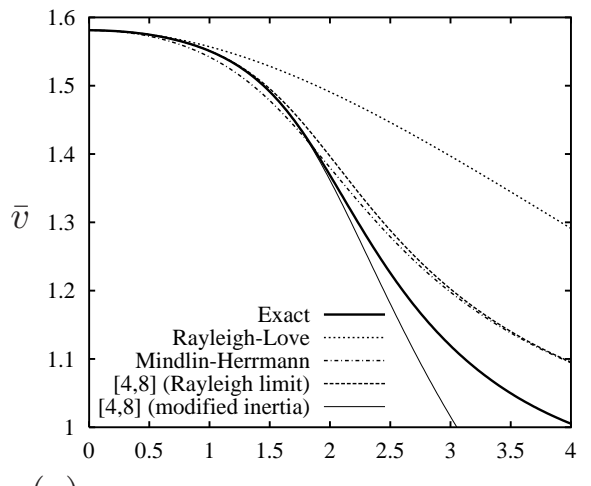

(a)

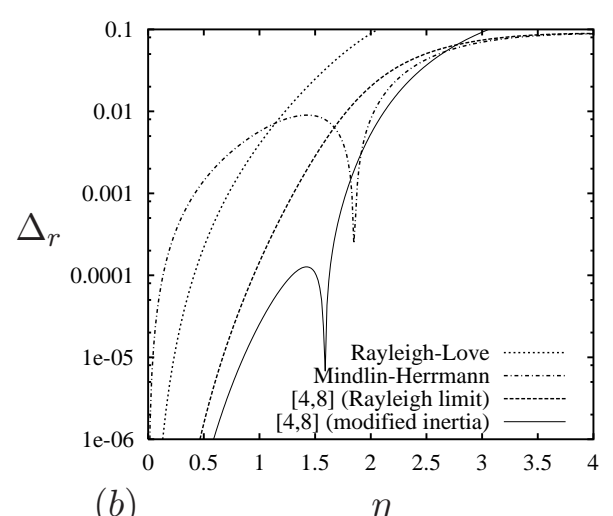

(b)

Fig. 5. Exact (thick solid) and approximate (thin solid, dotted and dashed) dispersion curves for the rod theories when $\nu=0.25$ : (a) phase velocity $\bar{v} \equiv v / c_{2}$ of harmonic waves against wave number; (b) relative approximation error against wave number.

It is easy to check that the low-frequency limit of the extraneous solution is $\eta^{2}=-1 / C_{1}$ and the long-wave high-frequency limit of the extraneous branch is $\bar{\omega}^{2}=-1 / C_{2}$. Thus, numerical stability requires $C_{1} \geqslant 0$; theories with $C_{2} \geqslant 0$ are not bandwidth-limited.

The original model $(45)$ is a $\langle 4,6\rangle$ theory with modified inertia. A $\langle 4,8\rangle$ theory with modified inertia may be obtained by selecting

$$
C_{1}=0, \quad C_{2}=-\frac{\phi(\nu)}{24\left(1-\nu^{2}\right)}, \quad \phi(\nu) \equiv 12 \nu^{4}+4 \nu^{3}-20 \nu^{2}-4 \nu+7,
$$

and is bandwidth-limited for positive $\nu$. Many engineering rod theories were derived with the assumption that their short-wave velocity limit equals the velocity of the Rayleigh wave $c_{R}$, as in exact Pochhammer-Chree dispersion relation. We may also achieve this by selecting

$$
\begin{gathered}
C_{1}=c_{R}^{2} \frac{\left(\phi(\nu) c_{R}^{2}-4 \nu^{3}+8 \nu^{2}+4 \nu-7\right)}{24\left(\nu^{2}-1\right)\left(1-c_{R}^{2}\right)^{2}}, \\
C_{2}=\frac{\left(24 \nu^{4}+4 \nu^{3}-32 \nu^{2}-4 \nu+7\right) c_{R}^{2}-\phi(\nu)}{24\left(1-\nu^{2}\right)\left(1-c_{R}^{2}\right)^{2}} .
\end{gathered}
$$

It is important to realise that any short-wave behaviour of a long-wave theory is equally meaningless. While the $\langle 4,8\rangle$ theory with parameters $(50)-(51)$ may produce better-looking dispersion curves in the short-wave limit, it also possesses extraneous solution terms, which may and will distort the solutions of boundary value problems unless special boundary conditions are formulated.

The behaviour of theory with parameters (50)-(51) is quite similar to that of the rod theory by Mindlin \& Herrmann [23]. It is easy to check that the Mindlin-Herrmann theory is not fourth order asymptotic and, consequently, cannot be represented in the form (47). By restricting the "tuning" parameters 
to $\kappa_{1}^{2}=1-\kappa^{2} / 2(\nu+1)$ it can be made a $\langle 4,6\rangle$ asymptotic theory. However, regardless of a particular choice of $\kappa$ and $\kappa_{1}$, the approximation accuracy of both $\langle 4,8\rangle$ theories with (49) and (50)-(51) is significantly better, which is demonstrated in Figure 5. The axisymmetric branch of the PochhammerChree dispersion relation is notoriously difficult to reproduce, and none of the considered theories quite manages to follow the fundamental mode in Figure 5(a). The plot of relative approximation errors in Figure 5(b) clearly demonstrates the benefits of using the $\langle 4,8\rangle$ theory with modified inertia (49). It may be re-written in terms of dimensional variables as

$$
c_{0}^{2} \frac{\partial^{2} u}{\partial x^{2}}=\left(1+\frac{R^{2}\left(4 \nu^{3}-8 \nu^{2}-4 \nu+7\right)}{24\left(1-\nu^{2}\right)} \frac{\partial^{2}}{\partial x^{2}}-\frac{R^{2} \phi(\nu)}{24 c_{0}^{2}\left(1-\nu^{2}\right)} \frac{\partial^{2}}{\partial t^{2}}\right) \ddot{u},
$$

and deserves to become the long-wave theory of choice for modelling rods.

\subsection{Bending of a thin plate}

For another example of asymptotic model for engineering structures we select a dynamic plate bending theory from [9]. A governing equation of the sixth order theory is given by

$$
D \Delta^{2} w+2 \rho h\left(1+h^{2} \frac{7 \nu-17}{15(1-\nu)} \Delta+h^{2} \frac{\left(422-424 \nu-33 \nu^{2}\right)}{1050(1-\nu) c_{2}^{2}} \frac{\partial^{2}}{\partial t^{2}}\right) \ddot{w}=0
$$

where $w$ is the mid-surface deflection, $D \equiv 2 E h^{3} / 3\left(1-\nu^{2}\right)$ the flexural rigidity, $E$ the Young modulus, $\nu$ the Poisson ratio, $c_{2} \equiv \sqrt{\mu / \rho}$ the shear wave speed, $\mu$ the shear modulus, $\rho$ the density, $h$ the plate half-thickness and the Laplace operator $\Delta=\partial^{2} / \partial x^{2}+\partial^{2} / \partial z^{2}$. This modified inertia theory reproduces three terms in the long-wave low-frequency expansion of the Rayleigh-Lamb dispersion relation for anti-symmetric modes, given by

$$
\begin{aligned}
\bar{\omega}^{2}=\eta^{4}- & \frac{7 \nu-17}{15(\nu-1)} \eta^{6}+\frac{62 \nu^{2}-418 \nu+489}{315(\nu-1)^{2}} \eta^{8} \\
& -\frac{\left(381 \nu^{3}-4995 \nu^{2}+14613 \nu-11189\right)}{4725(\nu-1)^{3}} \eta^{10}+O\left(\eta^{12}\right),
\end{aligned}
$$

in which $\bar{\omega} \equiv \sqrt{6(1-\nu)} \omega h / 2 c_{2}$ and $\eta \equiv k h$, see e.g. [22]. The asymptotic structure of (53) may be made more explicit by an appropriate non-dimensionalisation. Motivated by (54), we introduce

$$
x=\xi / k, \quad y=\zeta / k, \quad t=\frac{\sqrt{6(1-\nu)}}{2 c_{2} k \eta} \tau,
$$


and recast (53) in the form

$$
\frac{\partial^{2} w^{*}}{\partial \tau^{2}}+\Delta_{*}^{2} w^{*}+\frac{(7 \nu-17)}{15(1-\nu)} \frac{\partial^{2} \Delta_{*} w^{*}}{\partial \tau^{2}} \eta^{2}-\frac{\left(33 \nu^{2}+424 \nu-422\right)}{1575(1-\nu)^{2}} \frac{\partial^{4} w^{*}}{\partial \tau^{4}} \eta^{4}=0,
$$

with $w^{*}(\xi, \zeta, \tau) \equiv \eta^{2} l w(x, z, t)$ and $\Delta_{*} \equiv \partial^{2} / \partial \xi^{2}+\partial^{2} / \partial \zeta^{2} \cdot\langle 6,10\rangle$ theory (56) is different from all other theories we discussed so far in that its leading order is not hyperbolic. This results in the presence of asymptotic correction terms of varying orders, which reduces the number of options when formulating the class of asymptotically equivalent theories. To this end, we apply operators $\eta^{2} \Delta_{*}, \eta^{4} \Delta_{*}^{2}$ and $\eta^{4} \partial^{2} \partial \tau^{2}$, and obtain the superposition

$$
\begin{aligned}
\frac{\partial^{2} w^{*}}{\partial \tau^{2}}+ & \Delta_{*}^{2} w^{*}+\left(C_{1} \Delta_{*}^{3} w^{*}+\left(C_{1}+\frac{7 \nu-17}{15(1-\nu)}\right) \frac{\partial^{2} \Delta_{*} w^{*}}{\partial \tau^{2}}\right) \eta^{2} \\
+\left[C_{3} \Delta_{*}^{4} w^{*}+\right. & \left(C_{2}+C_{3}+C_{1} \frac{7 \nu-17}{15(1-\nu)}\right) \frac{\partial^{2} \Delta_{*}^{2} w^{*}}{\partial \tau^{2}} \\
& \left.+\left(C_{2}-\frac{33 \nu^{2}+424 \nu-422}{1575(1-\nu)^{2}}\right) \frac{\partial^{4} w^{*}}{\partial \tau^{4}}\right] \eta^{4}=0,
\end{aligned}
$$

where constants $C_{1}, \ldots, C_{3}$ satisfy one of the following conditions

$$
C_{1}^{2} \leqslant 4 C_{3} \quad \text { or } \quad \frac{C_{1}}{C_{3}}+\sqrt{\frac{C_{1}^{2}}{C_{3}^{2}}-\frac{4}{C_{3}}} \leqslant 0 .
$$

For the sake of brevity we only consider theories with modified inertia for which $C_{1}=C_{3}=0$. A slight improvement on (56) may be obtained by comparing the dispersion relation of (57) with (54) and matching the $O\left(\eta^{10}\right)$ term. Thus, a $\langle 6,12\rangle$ variant of $(54)$ requires

$$
C_{1}=C_{3}=0, \quad C_{2}=\frac{197 \nu^{2}-64 \nu+32}{1575(\nu-1)(7 \nu-17)} .
$$

It is instructive to compare the discussed asymptotic models with the popular Mindlin plate theory given by

$$
2 \rho h \frac{\partial^{2} w}{\partial t^{2}}+\left(\Delta-\frac{\rho}{\kappa^{2} \mu} \frac{\partial^{2}}{\partial t^{2}}\right)\left(D \Delta-\frac{2 \rho h^{3}}{3} \frac{\partial^{2}}{\partial t^{2}}\right) w=0,
$$

with the "shear coefficient" $\kappa^{2}$ to be chosen, see [1]. Similarly to the case of the Mindlin-Herrmann rod theory, this theory is not fourth order asymptotic, merely approximate. It can be made fourth order asymptotic by selecting $\kappa^{2}=5 /(6-\nu)$, see [24] and references therein. Mindlin suggested using "a compromise between" $\kappa^{2}=\pi^{2} / 12$ or $\kappa^{2}=c_{R}^{2} / c_{2}^{2}$, which are crude lower and upper bounds of the asymptotically correct value $\left(c_{R}\right.$ is the Rayleigh wave 


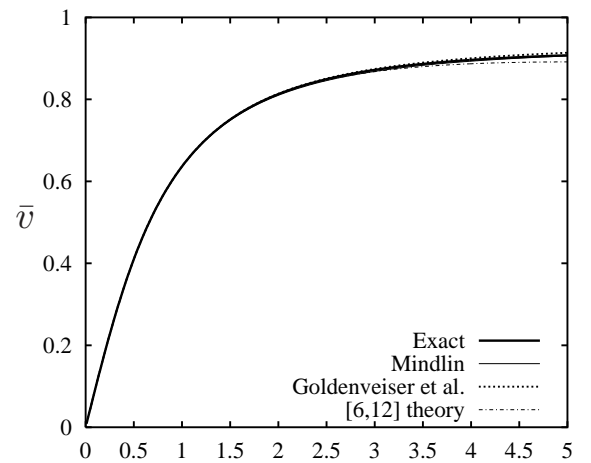

(a)

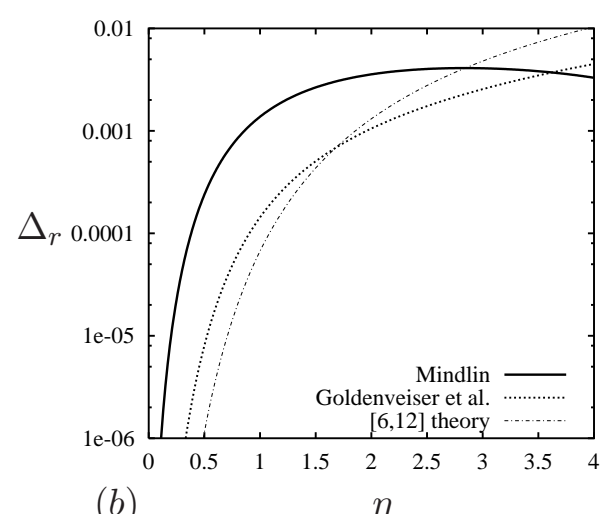

(b)

Fig. 6. Exact (thick solid) and approximate (thin solid, dotted and dashed) dispersion curves for the theories of plate bending when $\nu=0.25$ : (a) phase velocity $\bar{v} \equiv v / c_{2}$ of harmonic waves against wave number; (b) relative approximation error against wave number.

speed). In fact, if we select $\kappa^{2}=5 /(6-\nu)$ and rewrite (60) in terms of the non-dimensional variables used in (56), the result will only differ from (56) in the coefficient of $O\left(\eta^{6}\right)$ term. Specifically, instead of

$$
\frac{422-424 \nu-33 \nu^{2}}{1575(1-\nu)^{2}} \text { Mindlin's theory has } \frac{2(6-\nu)}{45(1-\nu)}
$$

which differ by no more then $5 \%$ for all positive Poisson ratios. Thus, Mindlin's theory may be recognised as a version of the asymptotically consistent governing equation (56) with slightly perturbed coefficients.

Our observations are supported by the dispersion curves in Figure 6. The plot in Figure 6(a) seems to demonstrate that all three theories (56), (57)-(59) and (60) are fairly accurate even in the range of moderate wave lengths. The Mindlin plate theory appears to be the most accurate, because its short-wave limit is fitted into the Rayleigh wave speed limit (we used $\kappa^{2}=c_{R}^{2} / c_{2}^{2}$ ). However, as is easily seen from Figure 6(b), Mindlin's model is significantly less accurate than both (56) and (57)-(59) within the range of its likely applications. It is also worth noting that the added asymptotic accuracy of the $\langle 6,12\rangle$ theory $(57)-(59)$ is actually reducing the range of accurate approximation, which confirms our considerations from Section 2.5.

Since there already exists a large number of numerical (finite element) implementations of the Mindlin theory, it seems appropriate to suggest using the formally equivalent plate theory from [9] instead. This would only require to 
specify $\kappa^{2}=5 /(6-\nu)$ and use the modified governing equation

$$
\begin{aligned}
2 \rho h \frac{\partial^{2} w}{\partial t^{2}}+\left(\Delta-\frac{\rho}{\kappa^{2} \mu} \frac{\partial^{2}}{\partial t^{2}}\right)(D \Delta & \left.-\frac{2 \rho h^{3}}{3} \frac{\partial^{2}}{\partial t^{2}}\right) w \\
& =\frac{D\left(103 \nu^{2}-66 \nu-2\right)}{700 c_{2}^{2}} \frac{\partial^{4} w}{\partial t^{4}} .
\end{aligned}
$$

\section{Conclusion}

We demonstrated how relatively simple manipulations may significantly improve dynamic response of existing long-wave theories, which would be most relevant to stationary dynamics. Our procedure presents a natural way of "retrofitting" old asymptotic theories. The $\langle 4,8\rangle$ rod theory with modified inertia, obtained in Section 4.1 from the Rayleigh-Love governing equation, is, to our best knowledge, the most accurate fourth order rod theory available. While asymptotic models for bending cannot be significantly improved, we both explained the well-known numerical performance of Mindlin's plate theory and indicated useful modifications of it.

Our observations also demonstrate that it is usually possible to formulate theories that approximate governing equations more accurately than the other aspects of the problem, e.g. distributions of displacements and stresses across the thickness for plate and shell theories. Therefore, the dispersion relation cannot be considered a sole indicator of accuracy of the asymptotic theory.

Finally, we reiterate that our discussion does not touch upon the question of selecting appropriate boundary conditions for asymptotic governing equations. While the theories with modified inertia may be used without reformulating the boundary conditions, we demonstrated that this option is not generally available in the two-dimensional setting. Therefore, the problem of efficient derivation of the appropriate boundary conditions remains open and will become a subject of a separate investigation.

\section{References}

[1] R. D. Mindlin. Influence of rotatory inertia and shear on flexural motions of isotropic, elastic plates. Journal of Applied Mechanics (1951) 18 31-38.

[2] V. P. Smyshlyaev, K. D. Cherednichenko. On rigorous derivation of strain gradient effects in the overall behaviour of periodic heterogeneous media. Journal of the Mechanics and Physics of Solids (2000) 48(6-7) 1325-1357.

[3] R. E. Shafer. On quadratic approximation. SIAM Journal on Numerical Analysis (1974) 11(2) 447-460. 
[4] J. D. Kaplunov, L. Y. Kossovich, E. V. Nolde. Dynamics of Thin Walled Elastic Bodies. Academic Press, New York (1998).

[5] C. Kittel. Introduction to Solid State Physics. John Wiley \& Sons, Inc., New York (1996).

[6] C.-C. Wang, C. Truesdell. Introduction to Rational Elasticity. Noordhoff International Publishing, Leyden (1973).

[7] R. Courant, D. Hilbert. Methods of Mathematical Physics. Vol. II: Partial Differential Equations. John Wiley \& Sons, New York (1989).

[8] G. B. Whitham. Linear and Nonlinear Waves. John Wiley and Sons, New York (1974).

[9] A. L. Goldenveizer, J. D. Kaplunov, E. V. Nolde. On TimoshenkoReissner type theories of plates and shells. International Journal of Solids and Structures (1993) 30(5) 675-694.

[10] W. Chen, J. Fish. A dispersive model for wave propagation in periodic heterogeneous media based on homogenization with multiple spatial and temporal scales. Journal of Applied Mechanics (2001) 68(3) 153-161.

[11] A. Erdélyi. Asymptotic Expansions. Dover Publications Inc., New York (1987).

[12] A. V. Metrikine, H. Askes. One-dimensional dynamically consistent gradient elasticity models derived from a discrete microstructure. Part 1: Generic formulation. European Journal of Mechanics A/Solids (2002) 21(4) $555-572$.

[13] A. V. Metrikine. On causality of the gradient elasticity models. Journal of Sound and Vibration (2006) 297(3-5) 727-742.

[14] L. Brillouin. Wave Propagation in Periodic Structures. Dover Pulications, Inc., New York (1953).

[15] G. A. Baker, P. Graves-Morris. Padé Approximants. Part 1: Basic Theory. Addison-Wesley Publishing Co, London, Sydney, Tokyo (1981).

[16] I. V. Andrianov, J. Awrejcewicz. Continuous models for 1D discrete media valid for higher-frequency domain. Physics Letters A (2005) 345(1-3) 5562.

[17] J. P. Boyd. The devil's invention: Asymptotic, superasymptotic and hyperasymptotic series. Acta Applicandae Mathematicae (1999) 56(1) 1-98.

[18] R. Burridge, P. Chadwick, A. N. Norris. Fundamental elastodynamic solutions for anisotropic media with ellipsoidal slowness surfaces. Proceedings of the Royal Society of London, Series A (1993) 440(1910) 655-681.

[19] A. S. J. Suiker, A. V. Metrikine, R. de Borst. Comparison of wave propagation characteristics of the Cosserat continuum model and corresponding discrete lattice models. International Journal of Solids and Structures (2001) 38(9) 1563-1583.

[20] J. W. S. Lord Rayleigh. The Theory of Sound, vol. 1. Macmillan and Co, London (1877).

[21] A. E. H. Love. A Treatise on the Mathematical Theory of Elasticity. University Press, Cambridge (1927).

[22] K. F. Graff. Wave Motion in Elastic Solids. Dover Publications, Inc., 
New York (1991).

[23] R. D. Mindlin, G. A. Herrmann. A one-dimensional theory of compressional waves in an elastic rod. In Proceedings of the First U.S. National Congress of Applied Mechanics, 187-191 (1950) .

[24] N. G. Stephen. Mindlin plate theory: best shear coefficient and higher spectra validity. Journal of Sound and Vibration (1997) 202(4) 539-553. 